\title{
DOES PANDEIMIC PAVES WAY FOR INNOVATIONS IN HEALTHCARE?: A REVIEW
}

KEY WORDS: Pandemics, Medical innovations, Digital healthcare, COVID-19, Telemedicine

\section{Maanasa R*}

\section{Srinivas G}

Postgraduate student (MSc Public Health), Department of Epidemiology, The Tamil Nadu, Dr MGR Medical University, Chennai. *Corresponding Author

Professor and Head, Department of Epidemiology, The Tamil Nadu Dr MGR Medical University, Chennai.

\section{Sudha Seshayyan Vice Chancellor, The Tamil Nadu Dr MGR Medical University, Chennai.}

The world has witnessed innumerable epidemic and pandemic episodes for centuries from Small Pox to Influenza to Cholera and the most recent wave of COVID-19 still ruling global health. The contemporary healthcare and public health system witnessing every pandemic has been influenced by two trends over the past years, namely the development of scientific information about the etiology and means of disease control and the development of public recognition of disease control as both a probability and a public obligation. Also, every pandemic has urged the healthcare professionals and system to come up with innovative strategies to control and handle the disease. Like the Vaccination system after smallpox and Facemask after Influenza, every disease pandemic has thus left a new control strategy designed for them. The concept of Telemedicine is considered to be the latest innovation of the 21 st century and handful in-need during COVID-19 pandemic as it binds digital technology and healthcare. We, in this paper, have reviewed in detail, the innovative medical discoveries that have been essential in times of need.

\section{INTRODUCTION:}

With every new century or a new millennium, there has been a need for growth in the medical world, and scientists and healthcare professionals have understood this well enough to meet the challenge. There have been advancements in biological science that were accompanied by practical advancements like the invention of diagnostics and treatment care along with the slow evolution of public health and preventive medicine. This journey of evolution later led to various advancements significant to every pandemic attack around the world. (Kolčić, 2019)

Epidemics and Pandemics have ruled global healthcare for centuries with its repeating waves and courses causing devastating effects and has ravaged human civilizations. Going by the popular quote, "Necessity is the Mother of Invention", every pandemic raised the need for a new control strategy to contain its spread urging the public health professionals to launch innovations into the medical world. The untiring work of public health authorities towards scientific advancements, driven by cultural and social advances, transformed human lives for the better. After the endangering attack of Cholera, enhanced water, and sanitation technologies and disclosures have contributed to innovations in reducing the transmission of pathogens, as well as medications and vaccines. (Glenn McDonald, 2020) Thus, the Public health societies and their policies have in turn been dramatically shaped by the epidemics around the world.

\section{Pandemics and Medical advancements:}

Global healthcare has experienced a history of pandemics from years of $B C$ and $A D$ to the current era with major diseases like Influenza and Cholera holding greater influence over current medical and public health practice. However, when focussed on the post-pandemic period of every disease, scientists and healthcare professionals have come up with milestone advancements in control and prevention.

\section{1) Plague and Quarantine:}

The devastating "Black Death" attack of Plague all over Europe in the 14th Century claimed the lives of millions of people leaving very little without any affection. However, this pandemic has taught the medical world the concept of Quarantine and its effect in breaking the chain of infection.

Overwhelmed doctors and health authorities battling a catastrophic epidemic of bubonic plague in medieval Italy about 700 years ago had little knowledge about viruses or microbes, yet they knew the Black Death sufficiently to enact some of the world's first anti-contagion steps namely Isolation. (Dave Roos, 2020)

Isolating patients with a contagious disease from the unaffected for 40 days was described as Quarantine, a direct descendant of quarantino in Italy. It was first implemented as mandated by law stipulating that people from plagueinfested areas were not allowed to enter other districts unless they spend a month of isolation. As a result of this isolation, the healthcare professionals witnessed a remarkable understanding of the incubation period and more people were disease-free. (Dave Roos, 2020)

There were chances of societal breakdown, mass hysteria, or complacency with some form of danger that can be just as risky while implementing the new strategy, which later turned out to be one of the most successful methods of prevention of the spread of infectious diseases globally.

\section{2) Small Pox andVaccine:}

The origin of smallpox is believed to have affected mankind around 10,000 BC in areas around North-Eastern Africa, however, the earliest scientific evidence of a pandemic affection is traced back to the 16th century. The disease had a devastating effect on all sectors of society where on average, 3 out of 10 people affected died and those who survived were left with scars and believed to have developed immunity to the disease. (CDC, 2019)

The initial treatment against the disease was the intake of herbal remedies and the method for controlling its spread was variolation. Named after the variola virus (Smallpox), it was a process where a material, namely lancet is made wet with the fresh matter taken from a ripe pustule of a patient infected and this material is then subcutaneously rubbed over the arms or legs of an unaffected non-immune person. This method was expected to raise the immunity of people against the disease, which also occurred, resulting in lowering the case-fatality rate by ten times than natural smallpox affection. However, the process had its own negative effects, where many people died after the process. (Riedel, 2005) 
This result thus showed the need for an advanced methodology giving birth to the concept of vaccination, which was based on the process of variolation. Since variolation, the milkmaids who had gotten cowpox did not exhibit any smallpox symptoms, and Sir Edward observing this designed a human experiment based on the concept. Milkmaid Sarah Nelmes and James Phipps, the 9-year-old son of Jenner's gardener, were the first participants to examine this hypothesis. Dr. Jenner removed the substance from Nelmes 'hand from a cowpox sore and inoculated it onto Phipps' arm. Months later, Jenner regularly subjected Phipps to variola virus, but smallpox was never developed by Phipps. (CDC, 2019)

Further experiments followed, and in 1801, Jenner published his treatise "On the Roots of Vaccine Inoculation," in which he summarized his findings and expressed hope that the ultimate result of this activity would be the extinction of smallpox, the most terrible disease of the human race. The practice of variolation, which was widely accepted, was thus ultimately replaced by vaccination. The strain that was used to develop the vaccine for smallpox switched from cowpox to the vaccinia virus later at some point around the 1800 s.

\section{3) Influenza and Facemask:}

Pandemic episodes of Influenza are always threatening due to their catastrophic impact and relative unfamiliarity. The major attack of Spanish Flu in 1918 - 1919 resulted in more than 50 million deaths due to rapid disease spread and poor immune system. This raised the need for measures to combat the spread of infection, which were all around the world like wildfire, leading to the use of facemask to protect from its airborne effect.

However, the use of face masks were not new to the world as different types of masks have been used against various infections since the 17th century. Use of beak-like masks against plague, one-layered gauze mask for surgeons, and general hospital staff are few instances from the history. (Ruisinger, 2020) But in concern to public health, the use of face masks was established to be against respiratory infections and was thus made mandatory by law all over the world, when the spread of Influenza became viral.

The model of face masks kept evolving from thickly layered gauze masks to medical masks to reusable disposable paper and cloth masks with time and disease. This evolution highly favored public health officials to enforce its use during the influenza period among general people. Later, when the rate of spread decreased to the maximum, people started wearing them for a longer period than intended as they understood its purpose and effect. (Matuschek et al.,2020)

This period is still quoted as a period of successful public health strategy against air-borne infections like Tuberculosis and the current COVID-19 infection, resulting in the use of facemasks by law after a century. Thus, the dreadful pandemic Influenza paved the need and a way for an innovative medical control strategy that is a handful in need, especially in the current period.

\section{4) COVID-19 and Digital Healthcare:}

With the Pandemic wave continuing to affect thousands of people across countries since its first attack, there have been too many healthcare innovations (Figure 1) that the medical field has witnessed. Incorporating digital technology into a working system of healthcare using automated tools is the most important development brought out in response to this global health crisis.

COVID-19 pandemic has affected all sectors of society disrupting the framework of the economy, education, and most importantly healthcare. Shortage of Personal Protective Equipment (PPE), Healthcare workers incapacity, inadequate emergency wards, and hospital beds, Screening, Tracing, and tracking of patients have all been major hindrances that have been addressed adequately through advanced technology. (COVID-19 | Innovations in Healthcare, 2020)

Contact tracing, community awareness, and communication are the three major nightmares during every pandemic, which have been handled through software applications all over the world. Aarogya Setu app in India is one of the first and authentic apps approved by the Government of India which helped both public and government to learn and understand COVID, personal screening by the patient themselves, and contact tracing. (Aarogya Setu, 2020) Test for COVID-19 and patient monitoring have been made easy, reliable, and advance using 3D diagnostics by Artificial Intelligence (AI).

The Patient Status Engine (PSE) automates the collection of raw patient data and decision-support tools for clinicians, combining wearable sensors with wireless networks and big data to provide high-resolution patient monitoring. Installed behind hospital firewalls, this healthcare innovation includes wireless connections as part of its design, with patients connected through an Android "gateway" running Isansys applications that receive incoming data from sensors via doubly encrypted Bluetooth. High-dependency isolation wards can thus be created rapidly. (August $24 \&$ Jones, 2020)

Bridging Technology and Healthcare - The envisioned future: Global healthcare and global public health have come a long way since the first global pandemic yet we face new challenges that need to be addressed. One such unmet need is bridging advancing scientific technology into the working system of healthcare which is known to benefit both patients and physicians. One of the most effective ways to use existing technologies to facilitate optimal service delivery while minimizing the hazard of direct person-to-person exposure is the use of telemedicine.

Telemedicine refers to the process of digitally caring for patients where there is no direct contact between the physician and the patient. Using various advanced videoconferencing software, modern technology will help doctors to consult patients in the most stable and quick to use method. Thus, at the time of any epidemic or pandemic condition, telemedicine has the potential to improve the research of epidemiological, control of disease, and management of clinical cases. (Portnoy et al., 2020)

While this concept has been proposed for years, it has come up for consideration since COVID-19 as part of contact prevention and still has few areas to be addressed. This involves the need to evaluate its efficacy over older people who are high-risk persons in the community in various health fields, especially in the field of home nursing. As it does not include in-person appointments, it is also strongly recommended to investigate its approach in the area of psychiatry. Other future studies will be able to tap into evaluating the satisfaction of patients and healthcare providers with telehealth services and their potential (Monaghesh \& Hajizadeh, 2020)

INNOVATIONS DURING COVID-19
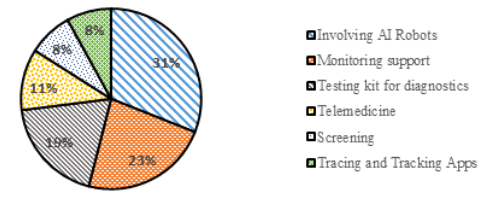

\section{CONCLUSION:}

Healthcare has been evolving for ages especially after every pandemic with the challenge posed upon the global public health and this trend continues even during the current COVID-19 situation. The introduction of the concept of public 
health, quarantine, vaccine, face mask, and a lot more have all been consequences of global epidemics and pandemics to meet the need. Thus in today's era, the concept of digital technology in healthcare and telemedicine has evolved and been discussed during COVID-19, which is a promising innovation in healthcare. This however still needs to be explored further for better results in the upcoming future.

\section{DECLARATIONS:}

Compliance with Ethical standards: This article complies with all the ethical standards of the journal and does not contain any study with human or animal subjects performed by any of the authors.

\section{Conflict of Interest: Nil}

Role of funding source: No funding source

Ethical Approval: Not applicable

Informed Consent: Not applicable

\section{References:}

1. Aarogya Setu. (2020). Retrieved October 18, 2020, from https://aarogyasetu. gov.in/

2. August 24, \& Jones, 2020 Rachel. (2020, August 24). 10 healthcare innovations in the fight against COVID. Raconteur. https://www.raconteur.net/ healthcare/healthcare-innovation-covid/

3. CDC.(2019, February 15). History of Smallpox | Smallpox | CDC.https://www. cdc.gov/smallpox/history/history.htm

4. COVID-19 | Innovations in Healthcare. (2020). Retrieved October 13, 2020 from https://www.innovationsinhealthcare.org/covid-19-innovations-inhealthcare-responds/

5. Dave Roos. (2020). The Term "Quarantine" Comes From Medieval Efforts to Fight the Black Death-HISTORY. Retrieved October 13, 2020, from https://www.history.com/news/quarantine-black-death-medieval

6. Glenn McDonald. (2020). 5 Advances That Followed Pandemics-HISTORY. Retrieved October 13, 2020, from https://www.history.com/news/ pandemics-advances

7. Kolčić, I. (2019). History of major advances in medicine, social medicine and hygiene. 45.

8. Matuschek, C., Moll, F., Fangerau, H., Fischer, J. C., Zänker, K., van Griensven, M., Schneider, M., Kindgen-Milles, D., Knoefel, W. T., Lichtenberg, A., Tamaskovics, B., Djiepmo-Njanang, F. J., Budach,W., Corradini, S., Häussinger, D., Feldt, T., Jensen, B., Pelka, R., Orth, K., ... Haussmann, J. (2020). The history and value of face masks. European Journal of Medical Research, 25. https://doi.org/10.1186/s40001-020-00423-4

9. Monaghesh, E., \& Hajizadeh, A. (2020). The role of telehealth during COVID19 outbreak: A systematic review based on current evidence. BMC Public Health,20.https://doi.org/10.1186/s12889-020-09301-4

10. Portnoy,J.,Waller, M., \& Elliott,T. (2020).Telemedicine in the Era of COVID-19. The Journal of Allergy and Clinical Immunology. in Practice, 8(5), 1489-1491. https://doi.org/10.1016/j.jaip.2020.03.008

11. Riedel, S. (2005). Edward Jenner and the history of smallpox and vaccination. Proceedings (Baylor University. Medical Center), 18(1), 21-25.

12. Ruisinger, M. M. (2020). [The "Plague Doctor's Mask" in the German Museum for the History of Medicine, Ingolstadt]. NTM, 28(2), 235-252. https://doi.org/10.1007/s00048-020-00255-7 